\title{
MS03-P19 | Structural Determinants Of The Interaction Specificity At The G And Nc Interfaces Of Human Septins
}

Leonardo, Diego (Instituto de Física de Sao Carlos, Sao Carlos, BRA); Nascimento, André (Laboratório Nacional Luz Síncrotron CNPEM, Campinas, BRA); Valadares, Napoleao (Universidade de Brasília, Brasília, BRA); André, Ingemar (Lund University, Lund, SWE); Uson, Isabel (ICREA, Catalan Institution for Research and Advanced Studies, Barcelona, ESP); Garratt, Richard (Instituto de Física de Sao Carlos, Sao Carlos, BRA)

Septins are proteins involved in several cellular processes and to perform their functions, at least three of the four different groups must associate to form heterofilaments by forming two types of interface: The G and NC interface. Although the type of contact interface and the specific order of the septins in the assembly of the filament are known, the molecular mechanisms that control the correct polymerization of the filament are still unknown. Here, we describe studies performed at the $G$ and NC interfaces of septins in order to uncover the structural determinants of the interaction specificity. "G" combinations of septins were purified to be characterized using biophysical techniques, showing dimers in solution with the presence of GTP/GDP. The dimers exhibit a different thermal stability, which could indicate preferences during complex formation. This condition was studied with MST, demonstrating that there is no significant difference in the dissociation constants, which strengthens the idea of substitution between septins in the same group. In the "NC" interface studies, the structures of the coiled-coil in the septins 1,4 and 5 were solved. An antiparallel orientation was observed for SEPT1 and SEPT4, with a hydrophilic and a hydrophobic side (a novel motif not yet reported) whereas the coiled-coil of SEPT5 has a parallel orientation (with classic contact interface). This suggests that these sequences can adopt the two orientations, which could explain the formation of structures of high complexity such as networks of filaments which have been observed in vivo and in TEM images. 